\title{
Luteinizing Hormone and GATA4 Action in the Adrenocortical Tumorigenesis of Gonadectomized Female Mice
}

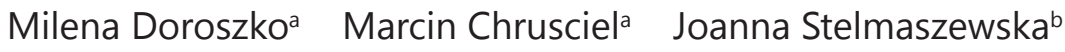 \\ Tomasz Slezak ${ }^{c}$ Adolfo Rivero-Mullerd,e Artur Padzik ${ }^{d}$ \\ Slawomir Anisimowicz ${ }^{f}$ Slawomir Wolczynski ${ }^{\text {b }} \quad$ Ilpo Huhtaniemia \\ Jorma Topparia,h Nafis A. Rahman ${ }^{a, b}$
}

aInstitute of Biomedicine, University of Turku, Finland; ${ }^{b}$ Department of Reproduction and Gynecological Endocrinology, Medical University of Bialystok, Poland; 'Department of Biochemistry and Molecular Biology, University of Chicago, Chicago, Illinois, USA; 'Turku Center for Biotechnology, Åbo Akademi and University of Turku, Finland; eDepartment of Biochemistry and Molecular Biology, Medical University of Lublin, Poland; 'Center of Gynecology and Reproductive Endocrinology Artemida, Bialystok, Poland; Institute of Reproductive and Developmental Biology, Imperial College London, London, UK; ' Department of Pediatrics, Turku University Hospital, Turku, Finland

\section{Key Words}

Lhcgr • GATA4 • Adrenocortical tumors • Molecular mechanisms • Transgenic mice

\begin{abstract}
Background/Aims: Physiological role of luteinizing hormone (LH) and its receptor (LHCGR) in adrenal remains unknown. In inhibin- $\alpha /$ Simian Virus $40 \mathrm{~T}$ antigen (SV40Tag) (inh $\alpha / \mathrm{Tag}$ ) mice, gonadectomy-induced (OVX) elevated LH triggers the growth of transcription factor GATA4 (GATA4)-positive adrenocortical tumors in a hyperplasia-adenoma-adenocarcinoma sequence. Methods: We investigated the role of LHCGR in tumor induction, by crossbreeding inha/Tag with Lhcgr knockout (LuRKO) mice. By knocking out Lhcgr and Gata4 in Ca1 adrenocortical cells (Lhcgr-ko, Gata4-ko) we tested their role in tumor progression. Results: Adrenal tumors of OVX inh $\alpha /$ Tag mice develop from the hyperplastic cells localized in the topmost layer of zona fasciculata. OVX inh $/$ Tag/LuRKO only developed SV40Tag positive hyperplastic cells that were GATA4 negative, cleaved caspase-3 positive and did not progress into adenoma. In contrast to Lhcgr-ko, Gata4-ko C $\alpha 1$ cells presented decreased proliferation, increased apoptosis, decreased expression of Inha, SV4OTag and Lhcgr tumor markers, as well as up-regulated adrenal- and down-regulated sex steroid gene expression. Both Gata4-ko and Lhcgr-ko C $\alpha 1$ cells had decreased expression of steroidogenic genes resulting in decreased basal progesterone production. Conclusion: Our data indicate that LH/LHCGR signaling is critical for the adrenal cell reprogramming by GATA4 induction prompting adenoma formation and gonadal-like phenotype of the adrenocortical tumors in inh $\alpha /$ Tag mice.
\end{abstract}




\section{Cellular Physiology Cell Physiol Biochem 2017;43:1064-1076

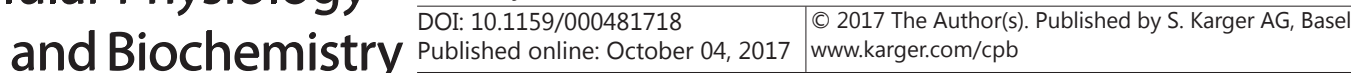

Doroszko et al.: LH Induced GATA4 Expression Causes Adrenal Tumors

\section{Introduction}

The embryonic adrenogonadal primordium (AGP) is a source of progenitors for three major steroidogenic organs: ovaries, testes and adrenal cortex [1]. AGP cells differentiate into gonadal and adrenal primordium and later into independent organs [1] although, recently AGP-like cells were found in the capsule of the mouse adrenal cortex [2]. Therefore disrupted endocrine homeostasis, e.g. due to gonadectomy (GDX) may promote the differentiation of AGP progenitors into neoplastic cells bearing gonadal-like phenotype [2]. Most of the wildtype (WT) mouse strains develop sub-capsular, non-steroidogenic, spindle-shaped A cells when aging [3,4]. Conversely, adrenal glands of gonadectomized certain inbred (DBA/2J, $\mathrm{NU} / \mathrm{J}$ ) and transgenic mice (21-OH-GATA-4, Inha ${ }^{-/}$) develop steroidogenically active adrenocortical tumors $[3,5,6]$. Therefore, they are referred to as mouse models that are genetically susceptible to gonadectomy-induced adrenocortical tumorigenesis (GIACT) [3, $5-8]$ and this phenotype is stronger in females than in males $[3,6,9]$.

The hallmark of neoplastic A cells and adrenocortical tumor cells is the expression of transcription factor GATA4 which does not occur in healthy adrenal cells $[3,6]$. On the other hand, GATA6, another member of the GATA transcription factor family, regulates adrenal steroidogenic cell differentiation [10]. Interestingly, the interplay between GATA6 and GATA4 transcription factors in adrenal pathophysiology has been suggested to determine the cell fate [10-13]. The lack of GATA6 in the adrenal gland promotes A cell formation [10], whereas GATA4 is pivotal for adrenal tumor onset in DBA/2J and 21-OH-GATA-4 mice [6, $14,15]$. Additionally, in $I n h a^{-/}$mouse model, it has been proposed that the development of tumorous and gonadal-like phenotypes of adrenal tumors is a consequence of the switch from GATA6 to GATA4 expression triggered by elevated gonadotropins [9].

Transgenic mice expressing the Simian Virus (SV40Tag) T-antigen under the inhibin- $\alpha$ promoter (inh $\alpha / \mathrm{Tag}$ ) [8] belong to the group of GIACT models. Both male and female transgenic mice present with gonadal tumors [16], but when prepubertally GDX they develop adrenocortical tumors in a hyperplasia-adenoma-carcinoma sequence by the age of 5-8 months $[8,17,18]$. In this model, genetic or chemical gonadotropin ablation prevents tumor formation [19], whereas transgenic elevation of LH results in the occurrence of gonadal and adrenocortical tumors [20]. An adrenocortical tumor cell line (C $\alpha 1$ ) has also been established from an inh $\alpha /$ Tag female founder [8] and extensively characterized [8, 17, 19]. Similar to other GIACT models, inh $\alpha /$ Tag adrenocortical tumors and C $\alpha 1$ cells express an array of gonadal factors including Lhcgr and transcription factor GATA4 (Gata4) [3, 8, 17, 21]. However, the direct involvement of LHCGR and GATA4 in the adrenocortical tumor induction, progression and phenotype, as well as their inter-relationship in these processes, remains unknown.

In the present study, we characterized the functional role of LHCGR and GATA4 expression in adrenocortical tumorigenesis in ovariectomized (OVX) inh $\alpha /$ Tag mice in vivo by crossbreeding them with LHCGR knockout mice (LuRKO), as well as in vitro by knocking out these genes in C $\alpha 1$ cells.

\section{Materials and Methods}

\section{Experimental animals}

Inh $\alpha$ /Tag females and control C57Bl/6N (WT) littermates were ovariectomized (OVX) at 21-24 days of age under isoflurane (2-4\%) anesthesia (Isoflo, Orion Pharma, Turku, Finland). Temgesic (buprenorphine, $0.1 \mathrm{mg} / \mathrm{kg} / 8 \mathrm{~h}$ ) (Schering-Plough, Brussels, Belgium) and Comforion® (ketoprofen, $5 \mathrm{mg} / \mathrm{kg} / 24 \mathrm{~h}$ ) (Orion, Turku, Finland) were administered subcutaneously as pre-operative and a post-operative analgesia respectively. 2-, 4-, 6-mo old intact and OVX, WT and inh $\alpha$ /Tag animals (n=8-14/group/for all the groups) were sacrificed by terminal cardiac puncture blood collection under isoflurane anesthesia. Tissues were weighted, adrenals snap-frozen in liquid nitrogen and/or fixed with 4\% paraformaldehyde (PFA). Blood was collected into a tube consisting $0.5 \mathrm{M}$ sterile EDTA solution, centrifuged at $3000 \mathrm{RPM}$ for 10 minutes in 


\section{Cellular Physiology Cell Physiol Biochem 2017;43:1064-1076 \begin{tabular}{l|l|l} 
and Biochemistry & $\begin{array}{l}\text { DOI: 10.1159/000481718 } \\
\text { Published online: October 04, } 2017\end{array}$ & $\begin{array}{l}\text { C) } 2017 \text { The Author(s). Published by S. Karger AG, Basel } \\
\text { www.karger.com/cpb }\end{array}$
\end{tabular}}

Doroszko et al.: LH Induced GATA4 Expression Causes Adrenal Tumors

$4{ }^{\circ} \mathrm{C}$ and plasma was stored in $-80^{\circ} \mathrm{C}$. To elucidate LHCGR involvement in the adrenal tumorigenesis in vivo, we crossbreed inh $\alpha /$ Tag mice with Luteinizing hormone Receptor Knockout (LuRKO) mice [22]. Samples from OVX: WT, inh $\alpha /$ Tag, LuRKO and inh $\alpha /$ Tag/LuRKO mice were collected the age of 7-mo as described above. Experimental mice were housed in a room with controlled light (12 h light and $12 \mathrm{~h}$ darkness) and temperature $\left(21 \pm 1^{\circ} \mathrm{C}\right)$ in a specific pathogen-free surrounding. Mice were fed with commercial mouse chow SDS RM-3 (Witham, Essex, UK) and tap water ad libitum. The State Provincial Office of Southern Finland approved all the animal experiments.

\section{Gene expression analysis}

Total RNA from cells and tissues was isolated using phenol/chloroform method with TRIsure (Bioline Reagents Ltd., London, UK) reagent according to the manufacturer's protocol. Prior to qPCR, 900 ng of total RNA was DNase I treated (\#18068015, Thermo Fisher Scientific, Waltham, MA) and transcribed (1 h in 48 ${ }^{\circ} \mathrm{C}$ ) using SensiFAST ${ }^{\mathrm{TM}}$ cDNA Synthesis Kit (\#BI0-65053, Bioline Reagents Ltd.). qPCR was carried out on a CFX Real Time PCR Detection System (Bio-Rad, Vienna, Austria), using the DyNAmo ${ }^{\mathrm{TM}}$ Flash SYBR ${ }^{\circledR}$ Green qPCR Kit (\#F415, Thermo Fisher Scientific) with 7.5ng of cDNA template in total reaction volume of 10 $\mu \mathrm{l}$ in duplicates. Conditions were as follows: $95{ }^{\circ} \mathrm{C}$ for $7 \mathrm{~min}$, $\left[95^{\circ} \mathrm{C}\right.$ for $15 \mathrm{~s}, 54-62{ }^{\circ} \mathrm{C}$ for $15 \mathrm{~s}, 72{ }^{\circ} \mathrm{C}$ for $15 \mathrm{~s}] \mathrm{x} 40,72{ }^{\circ} \mathrm{C}$ for $3 \mathrm{~min}, 65-95^{\circ} \mathrm{C}$ melt curve. Primer sequences are presented (for all online suppl. material, see www.karger.com/doi/10.1159/000481718) in supplementary Table S1. Gene expression was calculated using qBase MSExcel VBA applet [23]. In brief, Ct of gene of interest was normalized by geometric mean of 2-3 reference genes: peptidylprolyl isomerase A (Ppia), $\beta$-glucuronidase (Gusb), hypoxanthine phosphoribosyltransferase (Hprt1), peptidylprolyl isomerase B (Ppib) and hydroxymethylbilane synthase ( $\mathrm{Hmbs}$ ) validated separately for each experiment using Bio-Rad CFX Manager Software (Bio-Rad).

\section{Immunohistochemistry}

PFA fixed paraffin embedded adrenal glands from all experimental group females ( $n=4-6 /$ group) were cut into $4 \pm 1 \mu \mathrm{m}$ sections and immunohistochemically stained with antibodies and reagents listed (see supplementary material) in supplementary Table S2. Antigens were retrieved using high-temperature antigen retrieval method in citrate buffer (pH6), washed in TBS buffer with 0.1\% Tween20 (\#P1379, SigmaAldrich, Saint Louis, MO) and visualized using Liquid DAB+ Substrate Chromogen System (Dako, Glostrup, Denmark). Slides were scanned by Pannoramic 250 Slide Scanner (3DHISTECH Ltd., Budapest, Hungary).

\section{Semi-quantitative densitometric analysis}

Optical density of the antibody staining was calculated from minimum 5 images of good-quality representative areas of OVX inh $\alpha /$ Tag and OVX WT adrenals ( $n=4 /$ group). Images were acquired with Pannoramic Viewer (3DHISTECH Ltd.) at 50X magnification and analyzed by Fiji [24] using HematoxylinDiaminobenzidine (H-DAB) color deconvolution and DAB units of intensity (color 2) were counted. Optical density (OD) values were calculated using formula OD = $\log (255 /$ Mean intensity).

\section{Hormone measurement}

Plasma concentration of LH was measured by immunofluorometric (Delfia; Perkin Elmer, Turku, Finland) assay as described previously [25]. The intra- and inter- assay coefficients for LH were below 10\%. Plasma and cell culture medium progesterone were analyzed by Elecsys $®$ Progesterone II assay (Roche Diagnostics, Basel, Switzerland), using Cobas e411 immunoanalyzer (Roche Diagnostics). Detection limit for progesterone was $0.10 \mathrm{nmol} / \mathrm{l}$.

\section{CRISPR/Cas9 mediated knockout}

All-in-one pLV-U6gRNA-Ef1aPuroCas9GFP plasmids with targeting Lhcgr (MM0000334875, MM0000334894), Gata4 (MM0000234246, MM0000234282) and non-targeting guide sequence (\#CRISPR12-1EA, control) were obtained as custom-made plasmids from Sigma- Aldrich. Packaging cell line 293FT was transfected with a mixture of $2.6 \mu \mathrm{g}$ of plasmid and $26 \mu \mathrm{l}$ of MISSION® Lentiviral Packaging Mix (Sigma- Aldrich) using FuGENE® 6 Transfection Reagent (\#E2691, Promega) according to the provided protocol. After $72 \mathrm{~h}$, viral particles were concentrated for $2 \mathrm{~h}$ in $25.000 \mathrm{rpm}$ and snap frozen in liquid nitrogen. C $\alpha 1$ cells were seeded 50000 cells/well on 24-well plate and let to attach over-night. Next day, lentiviral stocks were added in low volume of full media and incubated for $16 \mathrm{~h}$. Single GFP positive cells were isolated by fluorescence-activated cell sorter (FACS) (BD FACSaria II, Becton Dickinson, Franklin Lakes, NJ) into microtiter plates to establish clonal cell populations.

\section{KARGER}




\section{Cellular Physiology Cell Physiol Biochem 2017;43:1064-1076 \begin{tabular}{l|l} 
and Biochemistry Published online: October 04, 2017 & $\begin{array}{l}\text { C } 2017 \text { The Author(s). Published by S. Karger AG, Basel } \\
\text { www.karger.com/cpb }\end{array}$ \\
\hline
\end{tabular}}

\section{Genotyping of knockout clones}

Clones were cultured in 10\% FCS medium, and genotyped using qPCR (primer sequences, see supplementary material, Table S1). Amplicons were separated by electrophoresis on 4\% agarose gels (\#17856, Thermo Fischer Scientific) and amplicons with significant size difference were isolated from the gel (\#28704, QIAquick Gel Extraction Kit, Qiagen) and Sanger sequenced (The Finnish Microarray and Sequencing Centre, Turku, Finland). Three mutated clones for Lhcgr and Gata4 were selected for further experiments (see supplementary material, sequences in Suppl. Fig. S3) and analyzed separately.

\section{In vitro experiments}

Mycoplasma-free C $\alpha 1$ murine adrenocortical cell line [8], and 3 mutated clones/gene were cultured in phenol red-free DMEM F-12 medium (Sigma- Aldrich) supplemented with 10\% heat-inactivated fetal calf serum (FCS) and $5 \mathrm{U} / \mathrm{ml}$ of penicillin/streptomycin (\#15140-122, Gibco) (full culture medium). Results from 3 mutated clones for Lhcgr (Lhcgr-ko) or Gata4 (Gata4-ko) were pooled and compared with the Cas 9 expressing C $\alpha 1$ cells (mock).

Cell proliferation rate was analyzed using CyQUANT® Cell Proliferation Assay kit (C7026, Life Technologies) in 8-plicates according to manufacturer's protocol. In brief, C $\alpha 1$ (passages 7, 11, 20), mock and ko cells were seeded on 96 well plates, 5000 cells/well in DMEM/F12 supplemented with 0.5\% steroid stripped serum (SFS). One million cells of each cell line was harvested to prepare a cell line-specific DNA standard curve. Cells were grown in medium containing $2.5 \%$ SFS without or supplemented with human recombinant hCG (50ng/ml) for $48 \mathrm{~h}$ (Serono, Geneva, Switzerland). Fluorescence signal was read using Wallac 1420 Victor2 Microplate Reader (Perkin Elmer, Turku, Finland). Standard curves and interpolated values were obtained using GraphPad Prism.

Caspase 3/7 activity was assessed using Caspase Glo 3/7 kit (Promega) according to the provided protocol. In brief, cells were seeded 8000/well on 96 well plate and assay was performed after $16 \mathrm{~h}$ of incubation. Absolute luminescence was normalized by $\mathrm{C} \alpha 1$-mock values.

For progesterone production measurements cells were seeded on 24-well plates (80000/well) in phenol red-free DMEM F-12 medium supplemented with $0.5 \%$ SFS. Medium was collected after $24 \mathrm{~h}$ and stored at $-8{ }^{\circ} \mathrm{C}$ until the analysis.

For the measurement of cAMP production cells were seeded on 24-well plates (120000/well) in medium containing $0.5 \%$ SFS and allowed to attach over-night. Next day, medium was changed for medium containing $0.5 \%$ SFS without (C) or with $1,10,50 \mathrm{ng} / \mathrm{ml}$ of recombinant hCG or $1 \mu \mathrm{M}$ forskolin (FRK) (SigmaAldrich). Medium was collected after $1 \mathrm{~h}$ stimulation, mixed 1:1 with $2 \mathrm{mM}$ teophiline (Sigma- Aldrich), boiled for 5 minutes in a water bath and frozen in $-20^{\circ} \mathrm{C}$. Extracellular cAMP concentration was assessed by a standard radioimmunoassay method after acetylation according to the method of Harper and Brooker $[26,27]$. Pellet radiation was read using $\gamma$-counter and automatically subtracted to a standard curve (Wallac 1470 Wizard Gamma Counter, Perkin Elmer).

\section{Statistical analysis}

To test the statistical differences between 2 or more experimental groups Mann-Whitney U or KruskalWallis with multiple comparison of mean range as post hoc tests were used respectively. All numerical data were presented as mean \pm SEM. Graphs and statistical analysis were done with Graph Pad Prism 5 (GraphPad Software, San Diego California USA), values of $\mathrm{P}<0.05$ were considered to be significant.

\section{Results}

\section{Formation of the adrenocortical tumors in Inha/Tag mice}

Total adrenal (Fig. 1A) and tumor weights of OVX inh $\alpha /$ Tag females (Fig. 1B; calculated by subtraction of the average total adrenal weight of age-matched OVX WT from total adrenal weights of inh $\alpha /$ Tag mice) were significantly increased from 4-mo and continued at 6-mo vs. age-matched controls. Using histological and immunohistochemical staining criteria, two distinct types of neoplastic cells expressing SV40Tag were identified at 2-mo of age (Fig. 1C and see supplementary material Fig. S1A). The first abnormal cells in the intact and OVX inh $\alpha$ /Tag mouse adrenals were the spindle-shaped neoplastic A cells (Fig. 


\section{Cellular Physiology and Biochemistry}

1D and see supplementary material Fig. S1C), originating from the sub-capsular region of the adrenal gland, and migrated towards the peri-medullary region. As observed in WT, these cells did not form tumors (see supplementary material, Fig. S1B, S1D). The second type of neoplastic cells observed had round nuclei (Fig. 1E), and was exclusively found in OVX inh $\alpha /$ Tag already at 2-mo, formed hyperplasia in the topmost layer of zona fasciculata, and resulted in a subsequent tumor formation. Both A and hyperplastic cells were SV40Tag, GATA4 and MKI67 positive (Fig. 1D-E). Representative pictures were taken from adjacent slides of the same specimen.

\section{Tumor cells lose GATA6 and gain GATA4 expression during their ontogeny}

The reciprocal expression of GATA6 and GATA4 in mouse models for adrenocortical tumorigenesis has been previously reported $[9,17]$, and was suggested to underpin the transition from adrenal-to-gonadal phenotype observed in these tumors [9]. We therefore examined the age-related GATA6 and GATA4 localization during the ontogeny of the adrenocortical tumors. GATA6 expression was abundant in the normal adrenal cortex of OVX

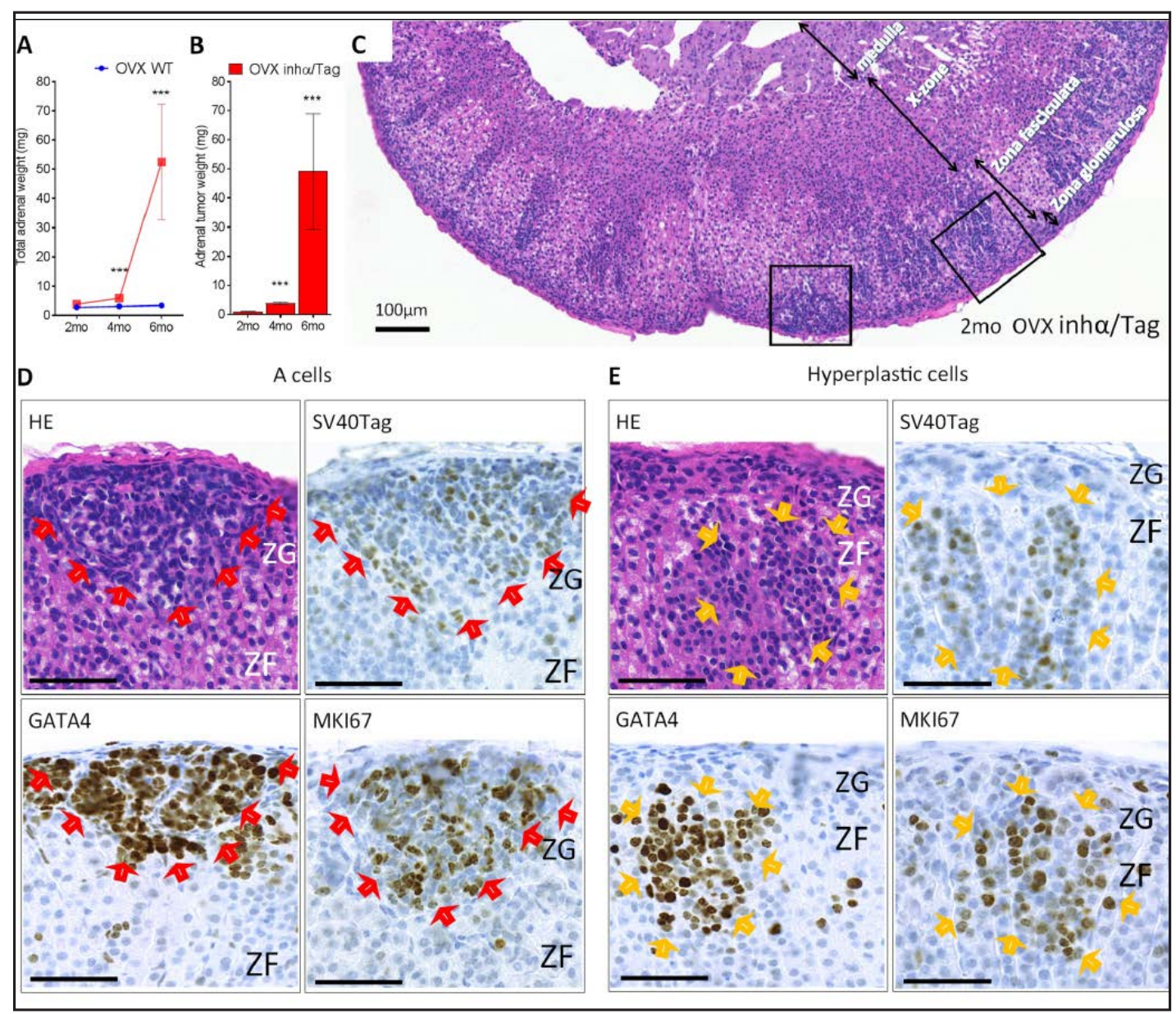

Fig. 1. Onset of adrenocortical tumors in inh $\alpha$ /Tag mice. (A) Age dependent adrenal gland mass of intact and OVX WT and inh $\alpha /$ Tag females (mean \pm SEM; ${ }^{* *}$. P $\leq 0.001 ; \mathrm{n}=8-14$ /group). (B) Approximate adrenal tumor weight calculated by average total adrenal weight of age-matched OVX WT subtracted from total adrenal weights of inh $\alpha /$ Tag mice (mean \pm SEM; ${ }^{* * *}$. P $\leq 0.001 ; n=8-14 /$ group). (C) Histopathology of the 2-month-old OVX female with the foci of (D) sub-capsular A cells and (E) hyperplastic cells stained for adrenal neoplastic cell biomarkers SV40Tag, GATA4 and proliferation marker MKI67 (n=4/group). Bar= $50 \mu \mathrm{m}$; ZG- zona glomerulosa, ZF- zona fasciculata, red arrows- A cells; yellow arrows- hyperplastic cells; OVX, subjected to prepubertal ovariectomy; WT, wild-type C57Bl/6N; inh $\alpha /$ Tag, transgenic mice expressing SV40Tag oncogene under inhibin $\alpha$ promoter. 
WT mice (Fig. 2A) and hyperplastic cells of 2-mo OVX inh $\alpha$ /Tag (Fig. 2B), and persisted in some cells within the tumor foci of 4-mo (Fig. 2C) and 6-mo (Fig. 2D) OVX inh $\alpha /$ Tag mice. In turn, GATA4 expression was absent in the normal adrenal cortex of OVX WT mice (Fig. 2F), with expression of GATA4 observed in hyperplastic cells of 2-mo OVX inh $\alpha$ /Tag (Fig. 2G), increasing in abundance within tumor foci of 4-mo (Fig. 2H) and 6-mo (Fig. 2I) OVX inh $\alpha$ / Tag mice. Densitometric analysis of the same region from consecutive sections (5 images/ specimen, $n=4$ / group), showed a significant decrease in GATA6 staining (Fig. 2E) in 4-mo and 6-mo OVX inh $\alpha /$ Tag vs. 6-mo OVX WT adrenals. Moreover, a concomitant increase in GATA4 staining was already present in hyperplastic cells at 2-mo, and continued to increase at 4-mo and 6-mo OVX inh $\alpha /$ Tag vs. 6-mo OVX WT (Fig. 2J).

Lack of LHCGR prevents GATA4 induction in SV40Tag-positive cells and adrenocortical tumor formation

To evaluate the role of LHCGR in the adrenocortical tumor induction in the OVX inh $\alpha$ / Tag model, we crossbred inh $\alpha /$ Tag mice with Lhcgr knockout (LuRKO) [22] mice (inh $\alpha /$ Tag/ LuRKO). At 7-mo of age, OVX inh $\alpha /$ Tag/LuRKO mice had significantly decreased total adrenal

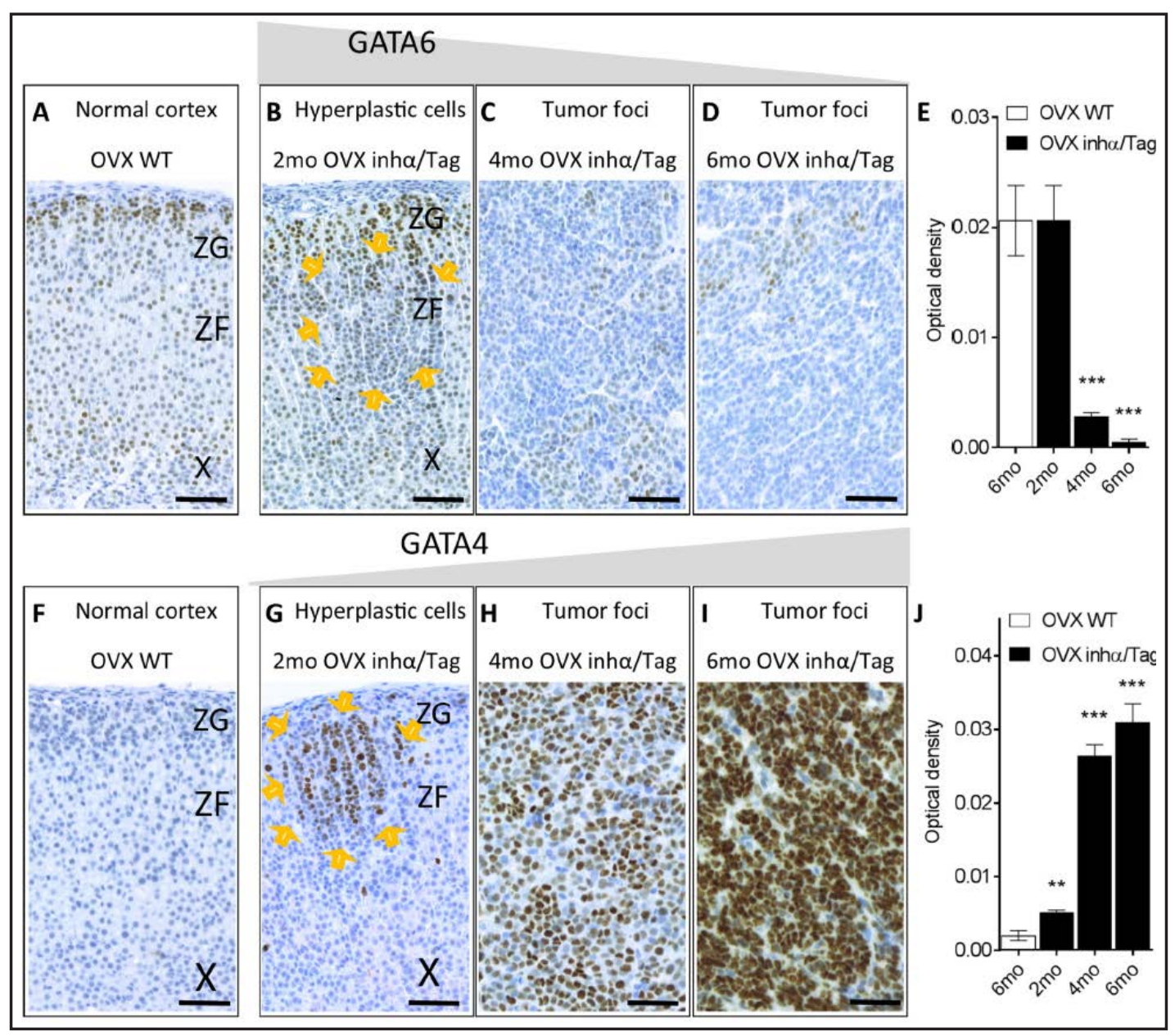

Fig. 2. GATA6 and GATA4 expression during the ontogeny of adrenocortical tumors in inh $\alpha /$ Tag mice. GATA6 localization in adrenals of 6mo OVX WT (A), hyperplastic cells of 2-mo (B), and in tumor foci of 4-mo (C) and 6-mo (D) OVX inh $\alpha /$ Tag mice (n=4/group). GATA4 localization in adrenals of 6-mo OVX WT (F), hyperplastic cells of 2-mo (G), and in tumor foci of 4-mo (H) and 6-mo (I) OVX inh $\alpha /$ Tag mice (n=4/group). Average optical density of GATA6 (E) and (GATA4) (J) staining in 6-mo OVX WT and 2-4-6-mo OVX inh $\alpha /$ Tag adrenals (mean \pm SEM; 5 images/ specimen, $\mathrm{n}=4$ / group; ${ }^{* *} . \mathrm{P} \leq 0.01$. ${ }^{* * *} . \mathrm{P} \leq 0.001$ ). Bar= 50 $\mu \mathrm{m}$; ZG- zona glomerulosa, ZF- zona fasciculata, $\mathrm{X}$ - $\mathrm{X}$-zone, yellow arrows- hyperplastic cells. 


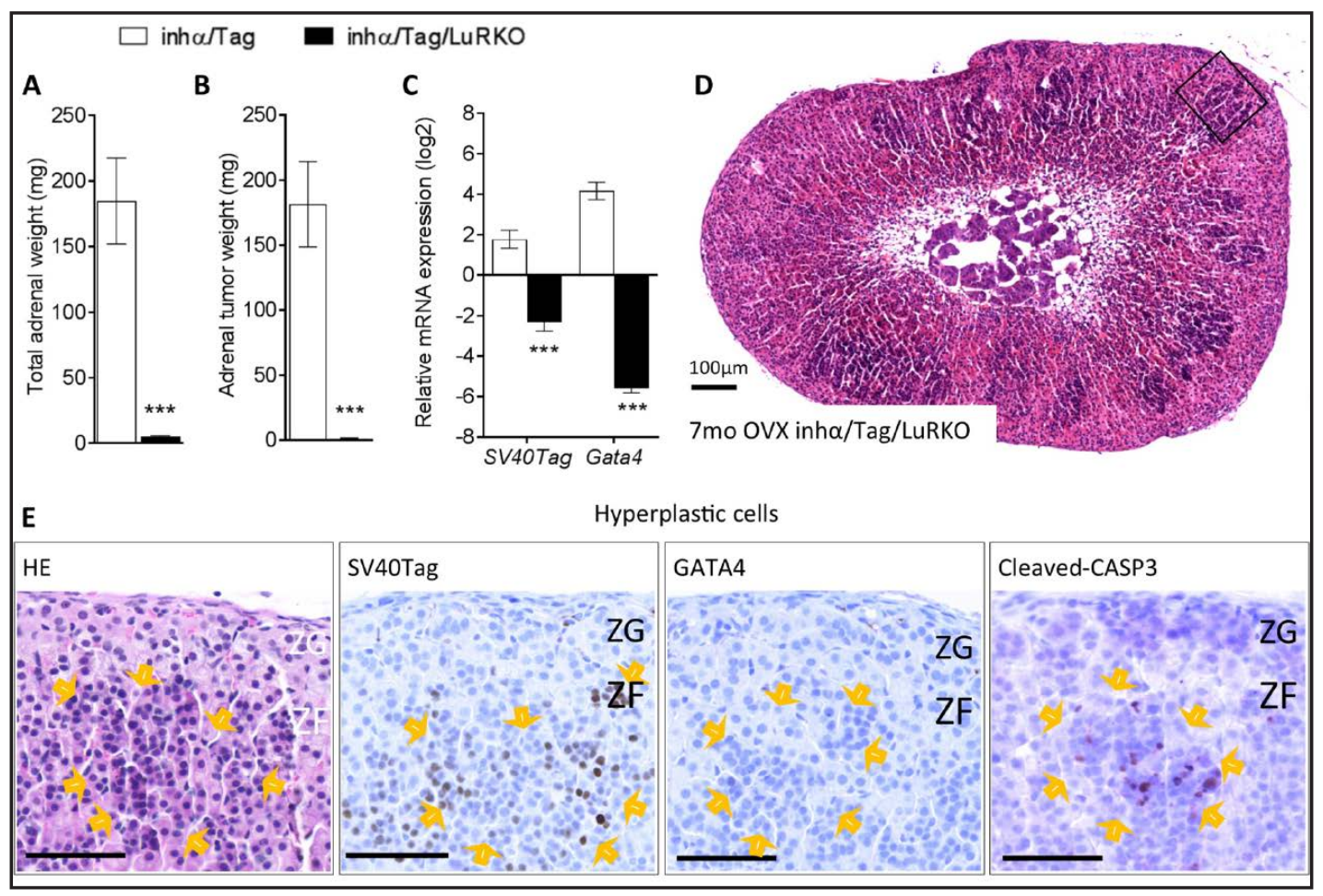

Fig. 3. Analysis of LH/LHCGR signaling in the onset of adrenal tumors in inh $\alpha /$ Tag mice. Comparison of adrenal (A), approximate adrenal tumor weights (B) and tumor marker SV40Tag, Gata4 expression (C) in

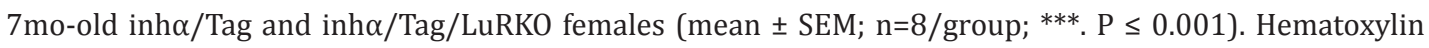
and eosin staining of 7mo-old OVX inh $\alpha$ /Tag/LuRKO adrenal gland (D), and localization of adrenocortical tumor biomarkers SV40Tag, GATA4 and Cleaved-CASP3 in hyperplastic cells (E) (n=6/ group). Bar= 50 $\mu$ m; ZG- zona glomerulosa, ZF- zona fasciculata, yellow arrows- hyperplastic cells; OVX, subjected to prepubertal ovariectomy; inh $\alpha /$ Tag, transgenic mice expressing SV40Tag oncogene under inhibin $\alpha$ promoter; inh $\alpha$ / Tag/LuRKO, inh $\alpha /$ Tag mice crossbred with LH deficient mice (LuRKO).

(Fig. 3A) and adrenal tumor weights (Fig. 3B), as well as down-regulated expression of the tumor markers SV4OTag and Gata4, in comparison to OVX inh $\alpha /$ Tag mice (Fig. 3C). Plasma concentrations of LH were similar (see supplementary material, Fig. S2A) in all the groups however, progesterone was increased in OVX inh $\alpha /$ Tag, and decreased in both OVX inh $\alpha$ / Tag/LuRKO and OVX LuRKO, when compared with OVX WT females (see supplementary material, Fig. S2B). The expression of neoplastic cell biomarker Gata4 was significantly decreased in OVX LuRKO mice vs. OVX WT (see supplementary material, Fig. S2C). As SV40Tag transcripts were detected in the adrenal glands of OVX inh $\alpha / \mathrm{Tag} / \mathrm{LuRKO}$ mice, we further analyzed their histopathology. The hematoxylin and eosin staining of 7-mo inh $\alpha$ / Tag/LuRKO adrenals (Fig. 3D) revealed the presence of hyperplastic cells (Fig. 3E), similar to those previously found in 2-mo inh $\alpha /$ Tag adrenals (Fig. 1E). We immunolocalized tumor biomarkers SV40Tag and GATA4, and to detect potential apoptotic cells, active caspase-3 (Cleaved-CASP3). Hyperplastic cells were SV40Tag and Cleaved-CASP3 positive, but GATA4 negative (Fig. 3E). Taken together, the absence of GATA4 in hyperplastic cells (Fig. 3E) could result in the decreased Gata4 expression in the adrenals (Fig. 3C) of 7-mo inh $\alpha /$ Tag/LuRKO mice.

GATA4 induces the tumorous phenotype, but both LHCGR and GATA4 regulate steroidogenesis in C $\alpha 1$ cells

We analyzed next the gene expression profile of Gata4 and Lhcgr markers, and steroidogenic enzymes in 6-mo OVX inh $\alpha /$ Tag and C $\alpha 1$ cells. Lhcgr and Gata4 expression were significantly increased in both 6-mo OVX inh $\alpha /$ Tag and C $\alpha 1$ cells when compared KARGER 


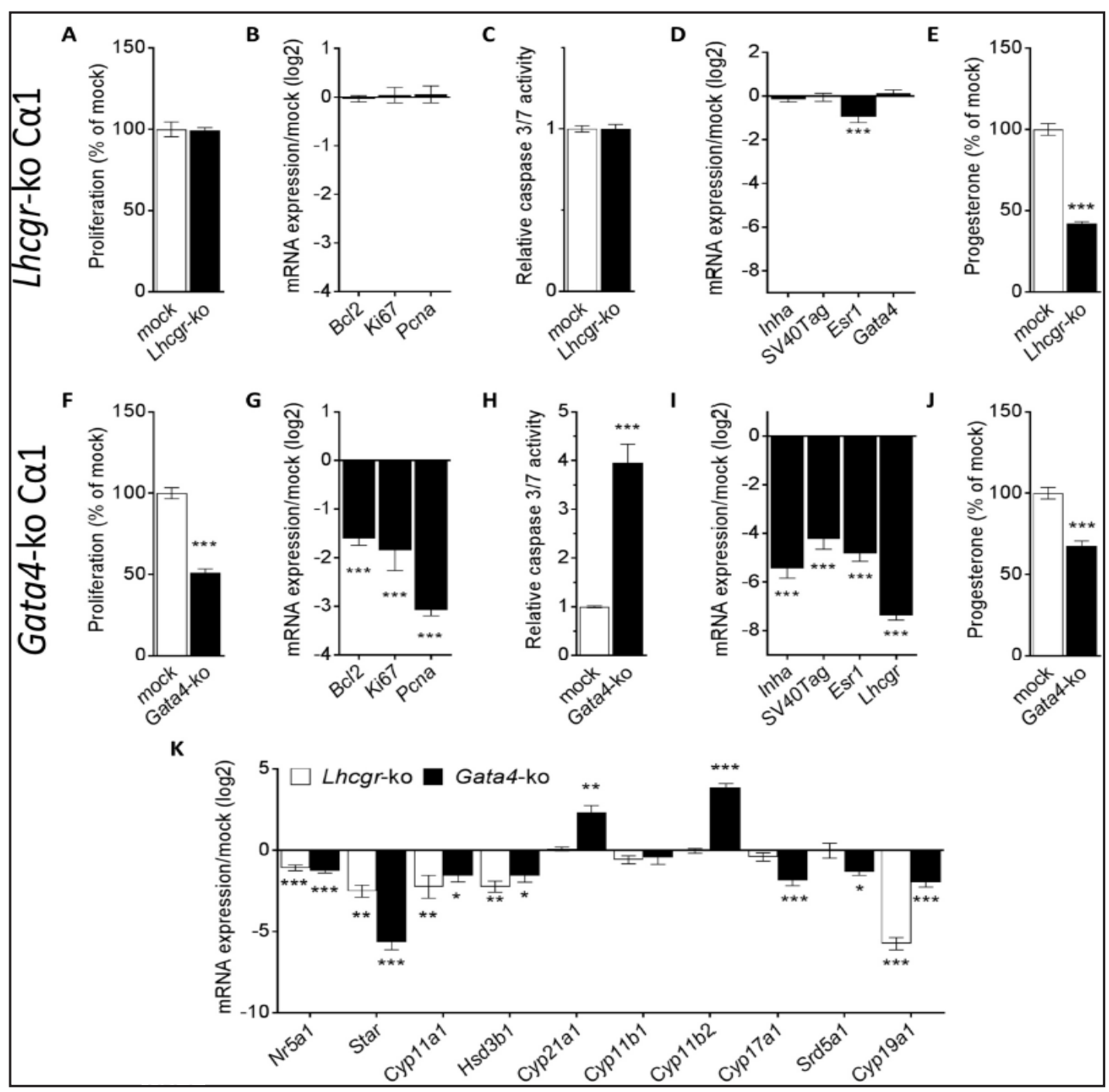

Fig. 4. Effects of Lhcgr and Gata4 knockout in C $\alpha 1$ cell line. Characterization of Lhcgr-ko and Gata4-ko cells by proliferation (A, F) gene expression of pro-survival Bcl2, proliferation markers Mki67 and Pcna (B, G), active caspase 3/7 levels (C, H), tumor cell markers Inha, SV40Tag, Lhcgr and Esr1 expression (D, I) and progesterone production (E, J) (mean \pm SEM; $n=5$-8/group). Comparison of steroidogenic enzymes gene expression in Lhcgr-ko and Gata4-ko cells vs. mock (K) (mean \pm SEM; n=9/ group). *. P $\leq 0.05$. **. P $\leq 0.01$. $* * *$. $\mathrm{P} \leq 0.001$.

to 6-mo OVX WT (see supplementary material, Fig. S3A). Moreover, C $\alpha 1$ cells expressed functional LHCGR as shown via the dose dependent hCG stimulation of cAMP production (see supplementary material, Fig. S3B). However, hCG stimulation did not affect C $\alpha 1$ cell proliferation (see supplementary material, Fig. S3C). Steroidogenic gene expression profiling showed up-regulation of the proximal Star (only in C $\alpha 1$ cells) and Cyp11a, up-regulation of gonad-specific Srd5a1 and Cyp19a1, and down-regulation of adrenal-specific Cyp21a1, Cyp11b1 and Cyp11b2 in comparison to OVX WT (see supplementary material, Fig. S3D).

To analyze the impact of LHCGR and GATA4 on tumor progression, we used CRISPR/ Cas9-mediated mutagenesis to knockout these genes in C $\alpha 1$ cells. Representative amplicon lengths of mutated clones as well as the actual sequences of Lhcgr and Gata4 mutants used in the experiments are shown in (see supplementary material) Fig. S4A-B and Fig. S4C-D, respectively. Functional LHCGR knockout was confirmed by abrogation of hCG-mediated cAMP production in all 3 independent Lhcgr-ko clones (see supplementary material, Fig. 
S4E). For all the experiments 3 different clones (see supplementary material, with mutations indicated in Fig. S4B and Fig. S4D) of Lhcgr-ko or Gata4-ko were used, results were pooled and compared with a mock-transfected cell line (control).

Lhcgr-knockout had no effect on either the proliferation of C $\alpha 1$ cells (Fig. 4A), expression of their anti-apoptotic Bcl2 and proliferation markers Pcna and Mki67 (Fig. 4B) or caspase 3/7 activity (Fig. 4C) in comparison to control. Gene expression analysis of the adrenocortical tumor biomarkers in Lhcgr-ko C $\alpha 1$ cells showed down-regulation of Esr1. In contrast, expression of Inha, SV40Tag and Gata4 remained unaffected (Fig. 4D). Gata4ko $\mathrm{C} \alpha 1$ cells had a significantly reduced proliferation rate vs. control (Fig. 4F) reflecting the decreased expression of anti-apoptotic Bcl2 and proliferation markers Mki67 and Pcna (Fig. 4G). Activity of caspase 3/7 was significantly increased in Gata4-ko Ca1 cells (Fig. 4H) indicating that these cells undergo apoptosis. Gene expression of tumor cell markers Inha, SV40Tag, Lhcgr and Esr1 was also decreased in Gata4-ko C $\alpha 1$ cells (Fig. 4I).

Basal progesterone production was significantly decreased in both Lhcgr-ko (Fig. 4E) and Gata4-ko (Fig. 4J) cells vs. mock. Moreover, lack of Lhcgr and Gata4 in Ca1 cells significantly down-regulated Nr5a1, Star, Cyp11a1, Hsd3ß1 and Cyp19a1 steroidogenic genes (Fig. 4K). Additionally, in Gata4-ko cells, adrenal steroidogenic enzymes Cyp21a1 and Cyp11b2 were up-regulated, while gonadal steroidogenic enzymes Cyp17a1 and Srd5a were down-regulated (Fig. 4K), showing enhanced adrenal steroidogenic phenotype.

\section{Discussion}

Adrenocortical tumor cells in inh $\alpha /$ Tag and $I n h a^{-/}$mice [7, 9] or large lipid-laden steroidogenic neoplastic B-cells in NU/J [5], DBA/2J [3] and 21-OH-GATA4 [6] appear after gonadectomy-induced elevated gonadotropins. It was previously considered that adrenocortical tumors in inh $\alpha /$ Tag mice, similarly to those of a complementary Inha ${ }^{-/}$model, originate from the $\mathrm{x}$-zone $[7,8]$. However, a recent report suggests that adrenocortical tumors in Inha $\%$ mice, as well as in other GIACT models, may originate from the sub-capsular pluripotent progenitors [9]. To revisit the origin of the inh $\alpha /$ Tag adrenocortical tumors we analyzed spatiotemporal expression of SV40Tag oncogene and identified the presence of two distinct types of SV40Tag positive cells, sub-capsular A and hyperplastic cells in adrenal parenchyma.

The tumor constituting SV40Tag expressing hyperplastic cells, at the age of 2-mo, were localized in the zona fasciculata, where a $6 \mathrm{~kb}$ promoter of Inha was shown to be most active in young and adult mouse adrenals [28]. Therefore, we suggest that the adrenocortical tumors in inh $\alpha /$ Tag model originate from zona fasciculata, not x-zone [8], nor from the stem/ progenitor cell niche [9] as previously suggested. Failure in tumor development in OVX inh $\alpha$ / Tag/LuRKO mice, indicate that SV40Tag expression alone is able to cause the hyperplasia but is not enough to drive tumor formation. Generally, SV40Tag expression inhibits p53 action and enables cells to pass the cell cycle despite DNA damage [29, 30], but may also require additional co-factors to maintain tumor formation [31]. An analogous situation was reported in a pancreatic tumor model expressing SV40Tag (RIP1-Tag2), where less than $2 \%$ of the islets expressing SV40Tag progressed into tumors [32]. In addition, anti-apoptotic BCL2 family members were identified as SV40Tag partners for tumor formation [31]. A recent study has shown [33], that expression of SV40Tag under a zona fasciculata-specific Akr1b7 promoter (AdTAg mice), resulted in a development of metastatic adrenal tumors in intact mice by the age of 8 months. Similarly to our model, SV40Tag expression was implicated mostly into tumor initiation, whereas spontaneously activated WNT/ $\beta$-catenin signaling promoted the malignant progression of adrenocortical tumors in intact mice [33]. In our OVX inh $\alpha /$ Tag/LuRKO model, lack of LHCGR and GATA4 expression in the hyperplastic cells resulted in a failure in hyperplasia to adenoma progression, suggesting these proteins as a potential SV40Tag partners during tumorigenesis.

Based on the in vitro studies, lack of GATA4 had more severe effects on the C $\alpha 1$ tumor cell phenotype, than the lack of LHCGR. In C $\alpha 1$ cell line, we found that GATA4 regulated 
proliferation and cell survival, as well as the expression of Inha, SV40Tag and Lhcgr. A recent report from our group showed that transgenic GATA4 expression under adrenalspecific 21-hydroxylase (21-OH) promoter (21-OH-GATA-4 model) was able to trigger tumor formation in $\mathrm{C57Bl} / 6$ strain mice, which is non-susceptible to GIACT [6]. However, similar to the inh $\alpha /$ Tag model, gonadectomy was a prerequisite for the tumor formation in 21-OH-GATA-4 mice [6]. On the other hand, knock out of GATA4 in mice susceptible to GIACT prevented tumor formation [14]. Moreover, GATA4 has been shown to be a potent driver of the tumorous and gonadal phenotype in the GIATC models $[6,9,14]$, as well as a pro-survival factor in gonadal Leydig and granulosa cells $[34,35]$. Therefore, GATA4 could be a potent partner for SV40Tag in tumor formation, regulator of anti-apoptotic pathways and gonad-related gene expression. The failure in hyperplasia-adenoma progression in OVX inh $\alpha /$ Tag/LuRKO mouse adrenals, supports the involvement of LH as an upstream factor for tumorigenesis in inh $\alpha /$ Tag mice $[9,20]$. In the complementary Inha ${ }^{-/}$model, folliclestimulating hormone (FSH) was proposed to trigger the tumorigenesis by induction of the GATA6 to GATA4 transcription factor switch [9]. In inh $\alpha /$ Tag model we see a gradual decrease of GATA6 expression with concomitant increase in GATA4 expression during the adrenal hyperplasia-adenoma transformation, suggesting that the tumorigenesis mechanism could be similar to that observed in Inha ${ }^{-/}$model.

The lack of effects of hCG or Lhcgr knock out on C $\alpha 1$ cell proliferation suggests that tumor progression is independent on LH/LHCGR signaling in this cell line model. Conversely, the tumor progression and survival was clearly regulated by GATA4. Moreover, no change was observed in Gata4 expression in Lhcgr-ko cells, suggesting that Gata4 expression is not regulated by LH/LHCGR, in the tumor cells. Therefore the high levels of Gata4 transcripts in adenoma and $\mathrm{C} \alpha 1$ cells might be an effect of GATA4 auto-regulation as previously shown [36]. Thus, LH/LHCGR signaling appears to be a prerequisite for the adrenocortical cell reprogramming probably through inducing the GATA6 to GATA4 switch, and thereafter GATA4 takes over the transcriptional control of the tumor cell progression.

Localization of SV40Tag in A cells of intact and OVX inh $\alpha /$ Tag mice supported the notion that oncogene expression is not sufficient for tumor formation. Moreover, the presence of A cells in OVX LuRKO and OVX inh $\alpha /$ Tag/LuRKO, confirmed an earlier report on their gonadotropin-independent appearance [6]. In contrast, a significant decrease of A cell biomarker Gata4 in OVX LuRKO and OVX inh $\alpha /$ Tag/LuRKO when compared to OVX WT adrenals, indicated a decrease in their population when LH/LHCGR signaling was lacking. An increase in A cell population after GDX [37], and Lhcgr expression in a sub-population of A cells [21] suggest that LHCGR signaling may affect their proliferation.

Gene expression of gonadal-type steroidogenic enzymes was up-regulated at the expense of reduced adrenal steroidogenesis in the adrenal tumors of OVX inh $\alpha /$ Tag females and $\mathrm{C} \alpha 1$ cells. This corresponds to the impaired expression of steroidogenic enzymes in inh $\alpha /$ Tag males bearing adrenocortical tumors [21]. In vitro studies revealed that both Lhcgr and Gata4 are important regulators of general steroidogenic genes resulting in decreased progesterone production in Lhcgr-ko and Gata4-ko cells. Also, in vivo the absence of functional LHCGR resulted in a decreased plasma progesterone levels supporting the potent role of LHCGR in the steroidogenic activity of OVX mouse adrenal glands [38]. We and others [17], have shown that GATA4 regulates Lhcgr expression, therefore it is impossible to excise the direct and indirect (through Lhcgr regulation) effects of GATA4 on the general steroidogenic gene expression. In contrast, only in Gata4-ko cells, we saw a clear inversion of gonadal-like (Cyp17a1, Srd5a1, Cyp19a1) to adrenal (Cyp21a1, Cyp11b2) steroidogenic phenotype, consistent with the previous reports that gonadal steroidogenic phenotype in GIACT models is an effect of GATA4 expression $[9,14]$. Our data confirms that LHCGR is a potent steroidogenesis enhancer in the adrenal gland [38], however, GATA4 controls the gonadal steroidogenic phenotype in the adrenocortical tumor cells [9].

In conclusion, the gonadotropin-dependent tumorigenic mechanism in the adrenal gland of inh $\alpha /$ Tag mice is activated in the cells of zona fasciculata that express GATA6. Increased LH/LHCGR signaling in definitive adrenocortical cells causes a transcription factor switch 


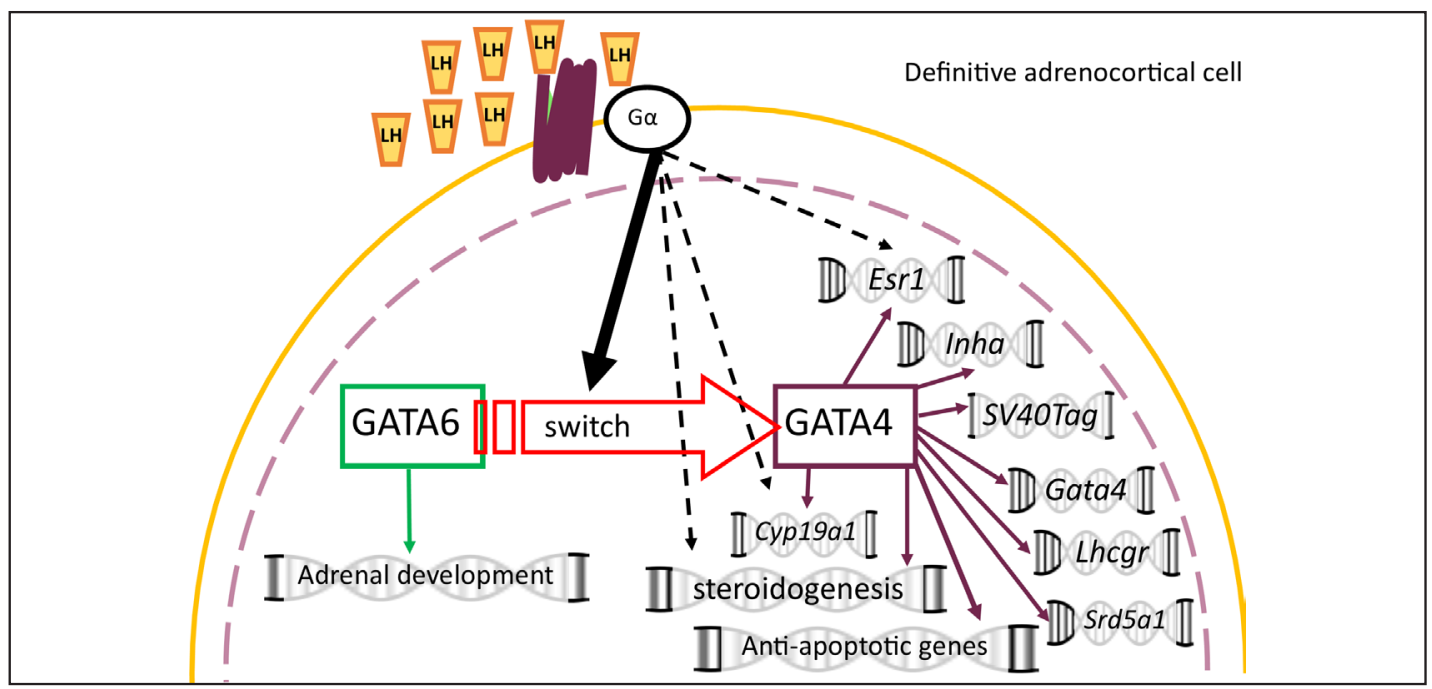

Fig. 5. Possible mechanism of SV40Tag oncogene activation in inh $\alpha /$ Tag adrenals. Oncogene expression in our inh $\alpha$ /Tag model is dependent on the activation of inhibin $\alpha$ promoter. Gonadectomy-induced chronically elevated LH levels up-regulate the expression of LHCGR, and LH/LHCGR signaling induces expression of GATA4, which independently of gonadotropins, takes control on the regulation of inhibin $\alpha$ promoter expression and cell survival.

from GATA6 to GATA4. GATA4 enhances strongly Inha/SV40Tag expression, the gonadal steroidogenic phenotype and most likely also stimulates the pro-survival pathways resulting in hyperplasia-adenoma transition. Once the transcription factor switch is induced, tumor progression becomes dependent on GATA4 and its downstream targets, but independent of LHCGR signaling (Fig. 5). Whether the process of GATA4 induction in human ACTs is similar to our inh $\alpha /$ Tag model, is an important question to address in future studies. Furthermore, identification of the direct GATA4 downstream targets in both inh $\alpha /$ Tag and human ACTs could be of great importance to find potential therapeutic targets for human ACTs.

\section{Abbreviations}

A cells (sub-capsular spindle shaped neoplastic non-steroidogenic type 'A' cells); B cells (lipid laden neoplastic steroidogenic type 'B' cells); SV40Tag (Simian Virus 40T

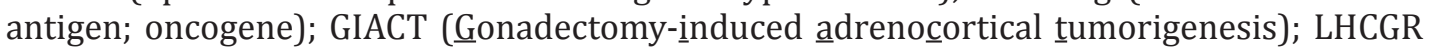
(luteinizing hormone receptor);

\section{Acknowledgements}

We would like to thank Dr Kim Jonas, St George's University of London, for the English language check of the revised manuscript; Taina Kirjonen from the Department of Physiology; Erica Nyman, Marja-Riitta Kajaala, Heli Niittymäki from the Turku Center for Disease Modeling (TCDM); The Finnish Microarray and Sequencing Centre (FMSC), Viral Vector Facility; Ketlin Adel and Jouko Sandholm from the Cell Imaging Core at Turku Center for Biotechnology (BTK) for providing their technical assistance. This work was supported by Turku Doctoral Programme of Molecular Medicine (MD), Finnish Cultural Foundation (MD), Academy of Finland (NR, JT), Sigrid Juselius Foundation (JT), ERVA grant from Turku University Hospital (JT) and Polish National Science Center grant 2015/17/B/NZ5/00636. 


\section{Cellular Physiology Cell Physiol Biochem 2017;43:1064-1076 \begin{tabular}{l|l|l} 
and Biochemistry Published online: October 04, 2017 & $\begin{array}{l}\text { (c) } 2017 \text { The Author(s). Published by S. Karger AG, Basel } \\
\text { www.karger.com/cpb }\end{array}$
\end{tabular}}

\section{Disclosure Statement}

The authors have nothing to disclose.

\section{References}

1 Pihlajoki M, Dörner J, Cochran RS, Heikinheimo M, Wilson DB: Adrenocortical zonation, renewal, and remodeling. Front Endocrinol (Lausanne) 2015;6:27.

-2 Dörner J, Rodriguez VM, Ziegler R, Röhrig T, Cochran RS, Götz RM, Levin MD, Pihlajoki M, Heikinheimo M, Wilson DB: GLI1+ progenitor cells in the adrenal capsule of the adult mouse give rise to heterotopic gonadal-like tissue. Mol Cell Endocrinol 2017;441:164-175.

-3 Bielinska M, Parviainen H, Porter-Tinge SB, Kiiveri S, Genova E, Rahman N, Huhtaniemi IT, Muglia LJ, Heikinheimo M, Wilson DB: Mouse strain susceptibility to gonadectomy-induced adrenocortical tumor formation correlates with the expression of GATA-4 and luteinizing hormone receptor. Endocrinology 2003;144:4123-4133.

4 Hofmann FG, Dickie MM, Christy NP: Studies of gonadectomized mice bearing adrenal cortical tumours. Acta Endocrinol (Copenh) 1960;34:84-96.

5 Bielinska M, Genova E, Boime I, Parviainen H, Kiiveri S, Leppaluoto J, Rahman N, Heikinheimo M, Wilson DB: Gonadotropin-induced adrenocortical neoplasia in NU/J nude mice. Endocrinology 2005;146:39753984.

6 Chrusciel M, Vuorenoja S, Mohanty B, Rivero-Müller A, Li X, Toppari J, Huhtaniemi I, Rahman NA: Transgenic GATA-4 expression induces adrenocortical tumorigenesis in C57Bl/6 mice. J Cell Sci 2013;126:1845-1857.

7 Matzuk MM, Finegold MJ, Mather JP, Krummen L, Lu H, Bradley A: Development of cancer cachexia-like syndrome and adrenal tumors in inhibin-deficient mice. Proc Natl Acad Sci U S A 1994;91:8817-8821.

-8 Kananen K, Markkula M, Mikola M, Rainio EM, McNeilly A, Huhtaniemi I: Gonadectomy permits adrenocortical tumorigenesis in mice transgenic for the mouse inhibin alpha-subunit promoter/simian virus 40 T-antigen fusion gene: evidence for negative autoregulation of the inhibin alpha-subunit gene. Mol Endocrinol 1996;10:1667-1677.

-9 Looyenga BD, Hammer GD: Origin and identity of adrenocortical tumors in inhibin knockout mice: implications for cellular plasticity in the adrenal cortex. Mol Endocrinol 2006;20:2848-2863.

10 Pihlajoki M, Gretzinger E, Cochran R, Kyronlahti A, Schrade A, Hiller T, Sullivan L, Shoykhet M, Schoeller EL, Brooks MD, Heikinheimo M, Wilson DB: Conditional mutagenesis of Gata6 in SF1-positive cells causes gonadal-like differentiation in the adrenal cortex of mice. Endocrinology 2013;154:1754-1767.

11 Rohrig T, Pihlajoki M, Ziegler R, Cochran RS, Schrade A, Schillebeeckx M, Mitra RD, Heikinheimo M, Wilson DB: Toying with fate: Redirecting the differentiation of adrenocortical progenitor cells into gonadal-like tissue. Mol Cell Endocrinol 2015;408:165-177.

12 Padua MB, Jiang T, Morse DA, Fox SC, Hatch HM, Tevosian SG: Combined loss of the GATA4 and GATA6 transcription factors in male mice disrupts testicular development and confers adrenal-like function in the testes. Endocrinology 2015;156:1873-1886.

-13 Tevosian SG, Jiménez E, Hatch HM, Jiang T, Morse DA, Fox SC, Padua MB: Adrenal Development in Mice Requires GATA4 and GATA6 Transcription Factors. Endocrinology 2015;156:2503-2517.

-14 Krachulec J, Vetter M, Schrade A, Lobs AK, Bielinska M, Cochran R, Kyronlahti A, Pihlajoki M, Parviainen H, Jay PY, Heikinheimo M, Wilson DB: GATA4 is a critical regulator of gonadectomy-induced adrenocortical tumorigenesis in mice. Endocrinology 2012;153:2599-2611.

15 Johnsen IK, Slawik M, Shapiro I, Hartmann MF, Wudy SA, Looyenga BD, Hammer GD, Reincke M, Beuschlein F: Gonadectomy in mice of the inbred strain CE/J induces proliferation of sub-capsular adrenal cells expressing gonadal marker genes. J Endocrinol 2006;190:47-57.

16 Kananen K, Markkula M, Rainio E, Su J, Hsueh A, Huhtaniemi IT: Gonadal tumorigenesis in transgenic mice bearing the mouse inhibin alpha-subunit promoter/simian virus T-antigen fusion gene: characterization of ovarian tumors and establishment of gonadotropin-responsive granulosa cell lines. Mol Endocrinol 1995;9:616-627.

17 Rahman NA, Kiiveri S, Rivero-Muller A, Levallet J, Vierre S, Kero J, Wilson DB, Heikinheimo M, Huhtaniemi 


\section{Cellular Physiology Cell Physiol Biochem 2017;43:1064-1076 \begin{tabular}{l|l|l} 
and Biochemistry Published online: October 04, 2017 & $\begin{array}{l}\text { (c) } 2017 \text { The Author(s). Published by S. Karger AG, Basel } \\
\text { www.karger.com/cpb }\end{array}$
\end{tabular}}

I: Adrenocortical tumorigenesis in transgenic mice expressing the inhibin alpha-subunit promoter/simian virus 40 T-antigen transgene: relationship between ectopic expression of luteinizing hormone receptor and transcription factor GATA-4. Mol Endocrinol 2004;18:2553-2569.

18 Chrusciel M, Doroszko M, Stelmaszewska J, Li X, Ziecik AJ, Coelingh-Bennink HJ, Huhtaniemi I, Rahman NA: Transgenic mice expressing inhibin alpha-subunit promoter (inhalpha)/Simian Virus 40 T-antigen (Tag) transgene as a model for the therapy of granulosa cell-derived ovarian cancer. Reprod Biol 2014;14:25-31.

-19 Rilianawati, Paukku T, Kero J, Zhang FP, Rahman N, Kananen K, Huhtaniemi I: Direct luteinizing hormone action triggers adrenocortical tumorigenesis in castrated mice transgenic for the murine inhibin alphasubunit promoter/simian virus 40 T-antigen fusion gene. Mol Endocrinol 1998;12:801-809.

-20 Mikola M, Kero J, Nilson JH, Keri RA, Poutanen M, Huhtaniemi I: High levels of luteinizing hormone analog stimulate gonadal and adrenal tumorigenesis in mice transgenic for the mouse inhibin- $\alpha$-subunit promoter/Simian virus 40 T-antigen fusion gene. Oncogene 2003;22:3269-3278.

-21 Doroszko M, Chrusciel M, Belling K, Vuorenoja S, Dalgaard M, Leffers H, Nielsen HB, Huhtaniemi I, Toppari J, Rahman NA: Novel genes involved in pathophysiology of gonadotropin-dependent adrenal tumors in mice. Mol Cell Endocrinol 2017;444:9-18.

22 Zhang F-P, Poutanen M, Wilbertz J, Huhtaniemi I: Normal prenatal but arrested postnatal sexual development of luteinizing hormone receptor knockout (LuRKO) mice. Mol Endocrinol 2001;15:172-183.

23 Hellemans J, Mortier G, De Paepe A, Speleman F, Vandesompele J: qBase relative quantification framework and software for management and automated analysis of real-time quantitative PCR data. Genome Biol 2007;8:R19.

-24 Bernichtein S, Petretto E, Jamieson S, Goel A, Aitman TJ, Mangion JM, Huhtaniemi IT: Adrenal gland tumorigenesis after gonadectomy in mice is a complex genetic trait driven by epistatic loci. Endocrinology 2008;149:651-661.

25 Haavisto AM, Pettersson K, Bergendahl M, Perheentupa A, Roser JF, Huhtaniemi I: A supersensitive immunofluorometric assay for rat luteinizing hormone. Endocrinology 1993;132:1687-1691.

-26 Harper JF, Brooker G: Femtomole sensitive radioimmunoassay for cyclic AMP and cyclic GMP after 2'0 acetylation by acetic anhydride in aqueous solution. J Cyclic Nucleotide Res 1975;1:207-218.

27 Brooker G, Harper JF, Terasaki WL, Moylan RD: Radioimmunoassay of cyclic AMP and cyclic GMP. Adv Cyclic Nucleotide Res 1979;10:1-33.

28 Hsu S, Lai R, Nanuel D, Hsueh A: Different 5'-flanking regions of the inhibin-alpha gene target transgenes to the gonad and adrenal in an age-dependent manner in transgenic mice. Endocrinology 1995;136:55775586.

29 Levine AJ, Momand J, Finlay CA: The p53 tumour suppressor gene. Nature 1991;351:453-456.

-30 Hudson AL, Colvin EK: Transgenic Mouse Models of SV40-Induced Cancer. ILAR Journal 2016;57:44-54.

-31 Rogakou EP, Pilch DR, Orr AH, Ivanova VS, Bonner WM: DNA double-stranded breaks induce histone H2AX phosphorylation on serine 139. J Biol Chem 1998;273:5858-5868.

32 Bono AV, Salvadore M, Celato N: Gonadotropin-releasing hormone receptors in prostate tissue. Anal Quant Cytol Histol 2002;24:221-227.

-33 El Ghorayeb N, Bourdeau I, Lacroix A: Role of ACTH and other hormones in the regulation of aldosterone production in primary aldosteronism. Front Endocrinol (Lausanne) 2016;7:

34 Schrade A, Kyronlahti A, Akinrinade O, Pihlajoki M, Hakkinen M, Fischer S, Alastalo TP, Velagapudi V, Toppari J, Wilson DB, Heikinheimo M: GATA4 is a key regulator of steroidogenesis and glycolysis in mouse Leydig cells. Endocrinology 2015;156:1860-1872.

-35 Kyrönlahti A, Rämö M, Tamminen M, Unkila-Kallio L, Butzow R, Leminen A, Nemer M, Rahman N, Huhtaniemi I, Heikinheimo M: GATA-4 regulates Bcl-2 expression in ovarian granulosa cell tumors. Endocrinology 2008;149:5635-5642.

-36 Mazaud-Guittot S, Prud'Homme B, Bouchard MF, Bergeron F, Daems C, Tevosian SG, Viger RS: GATA4 autoregulates its own expression in mouse gonadal cells via its distal 1b promoter. Biol Reprod 2014;90:25.

-37 Bandiera R, Vidal VP, Motamedi FJ, Clarkson M, Sahut-Barnola I, Von Gise A, Pu WT, Hohenstein P, Martinez A, Schedl A: WT1 maintains adrenal-gonadal primordium identity and marks a population of AGP-like progenitors within the adrenal gland. Dev Cell 2013;27:5-18.

-38 Kero J, Poutanen M, Zhang FP, Rahman N, McNicol AM, Nilson JH, Keri RA, Huhtaniemi IT: Elevated luteinizing hormone induces expression of its receptor and promotes steroidogenesis in the adrenal cortex. J Clin Invest 2000;105:633-641. 